\title{
Prevalence and socio-demographic factors of SARS-CoV-2 antibody in multi-ethnic healthcare workers
}

\author{
Authors: Mehool Patel, ${ }^{\mathrm{A}}$ Meera Nair, ${ }^{\mathrm{B}}$ Eric Pirozzoli, ${ }^{\mathrm{C}}$ Marta C Cienfuegos ${ }^{\mathrm{D}}$ and Elizabeth Aitken ${ }^{\mathrm{E}}$
}

\begin{abstract}
Introduction
Healthcare workers are particularly susceptible to developing COVID-19 owing to close and frequent contact with COVID-19 patients. This cross-sectional study aimed to describe prevalence of SARS-CoV-2 antibodies among healthcare workers within a hospital trust and examine factors associated with increased prevalence of this antibody.
\end{abstract}

\section{Methods}

Data was obtained over a 4-week period in 2020 from a crosssectional prospective survey of healthcare workers serving a multi-ethnic inner-city population who had immunoglobulin G SARS-CoV-2 antibody test. Anonymised socio-demographic data about staff were cross-referenced with these tests.

Results

Of 7,013 staff, 6,212 (89\%) undertook the antibody test during this period. Overall detection rate was $26 \%(1,584 / 6,212)$. Univariate analyses revealed no differences in prevalence in terms of gender or age. Compared with white staff members (18\%), rates were higher in black (38\%) and Asian (27\%) members $(p<0.001)$. The rates in general wards $(43 \%)$ were higher compared with other areas; in emergency medicine and intensive care, prevalence was $23 \%(p<0.001)$. Regarding professional groups, prevalence was highest among nursing and allied clinical services (28\%), less in doctors (23\%) and lower in non-clinical staff (19\%).

Discussion

This large study has described prevalence of recent exposure to SARS-CoV-2 infection among healthcare workers and determined associations including ethnicity, professional groups and geographical areas within healthcare settings. This information will be useful in future COVID-19 studies examining the role of antibody testing both in general populations and in healthcare settings.

Authors: ${ }^{\mathrm{A}}$ consultant physician and deputy medical director, Lewisham and Greenwich NHS Trust, Lewisham, UK; ${ }^{B}$ chief people officer, Lewisham and Greenwich NHS Trust, Lewisham, UK; ${ }^{C}$ associate director of workforce, Lewisham and Greenwich NHS Trust, Lewisham, UK; Dhead of workforce intelligence, Lewisham

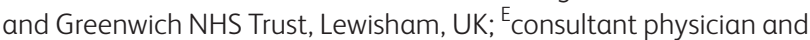
medical director, Lewisham and Greenwich NHS Trust, Lewisham, UK
KEYWORDS: COVID-19 antibody, prevalence, healthcare workers, SARS-CoV-2 antibody

DOI: 10.7861/clinmed.2020-0619

\section{Introduction}

Since the detection of the first cases of COVID-19 in Wuhan in December 2019, several laboratory tests have been developed to assist in its diagnosis. ${ }^{1,2}$ Tests include 'direct' tests that aim to detect the virus (SARS-CoV-2) itself and thereby detect 'current' illness as well as indirect antibody tests that aim to detect development of antibodies to the virus, thereby providing information on previous exposure to the virus. ${ }^{3-5}$ The infection with SARS-CoV-2 usually leads to seroconversion 11-14 days after the first symptoms. ${ }^{3-5}$

Previous studies that have examined the prevalence of SARSCoV-2 infection in healthcare workers have predominantly reported prevalence rates on the basis of direct tests or clinical symptoms, and in relatively smaller cohorts of healthcare workers. ${ }^{6-9}$ They were unable to examine any underlying sociodemographic factors, such as ethnicity, associated with SARSCoV-2 seroprevalence among these workers.

Healthcare workers are particularly susceptible to developing this illness owing to their close and frequent contact with COVID-19 patients. ${ }^{10,11}$ In order to provide information on the prevalence of COVID-19 in different regions of the country and help better understand how the disease spreads, particularly among healthcare workers within the NHS, NHS England promoted a nationwide antibody testing programme in May $2020 .^{12}$

The objectives of this cross-sectional study were to describe the prevalence of antibody to SARS-CoV-2 among healthcare workers (and thereby exposure to the virus) within a busy acute district general hospital trust and to examine factors that may be associated with increased prevalence of this antibody.

\section{Methods}

Data was obtained from a cross-sectional prospective survey of healthcare workers within a healthcare trust that employs 7,013 substantive staff members. The trust provides healthcare to a diverse multi-ethnic inner-city population from two extremely busy acute district general hospital legacy sites and several small community-based care centres. The community prevalence 
of COVID-19 in the area between April and May 2020 was significantly higher than the rest of the country. The tests were conducted over a 4-week period from 27 May 2020 until 24 June 2020.

Following guidance and availability of antibody testing for NHS staff by NHS England, the trust implemented a comprehensive trust-wide strategy to enable all its staff members to undertake the test. ${ }^{12}$ A COVID Antibody Implementation Group (CAIG) was set up that oversaw the development and implementation of this strategy. A standard operating procedure was developed to ensure a systematic structured operational plan was in place to ensure maximum uptake of the test. The group also developed a set of frequently asked questions and designed a consent form in accordance with guidance from NHS England. These were made available to the staff on the trust intranet.

The SARS-CoV-2 antibody test conducted was an immunoglobulin $\mathrm{G}$ (IgG) test developed and provided by Abbott laboratories. ${ }^{13}$

This SARS-CoV-2 IgG assay is a chemiluminescent micro-particle immunoassay (CMIA) intended for the qualitative detection of IgG antibodies to SARS-CoV-2 in human serum, serum separator tube and plasma (ACD, CPD, CPDA-1, dipotassium EDTA, tri-potassium EDTA, lithium heparin, lithium heparin separator tube, sodium citrate and sodium heparin). ${ }^{13}$ This assay detects IgG antibodies against the SARS-CoV-2 nucleocapsid protein.

Multiple, overlapping sources of notification were implemented to promote the availability of the test including regular twice weekly emails to all staff members, regular e-bulletins on the intranet, asking line managers to encourage their staff to consider the test, personal visits to all areas of the trust by members of the CAIG, and by verbal promotion by CAIG at every staff interaction. There were three principle methods by which the tests were made available to the staff: two designated areas (one at each legacy site) were identified that were fully staffed with multidisciplinary staff that included phlebotomists, physician associates, nurses and doctors that conducted the tests on a daily basis for up to
6 hours including weekends; mobile teams were also deployed to visit those areas of high vulnerability including emergency medicine departments, intensive care units, acute medical units and acute labour wards; and a community-based centre was also identified to enable those community staff members that were unable to attend the acute sites.

Informed consent was obtained from all staff members in accordance with NHS England guidance. The results of the test were conveyed to the staff via a mobile text (if they agreed to provide a contact number and consented to receiving the result) within 24-48 working hours.

For this study, anonymised data about the staff members was obtained from the Electronic Staff Record (ESR) held by the workforce department. This data was cross-referenced with anonymised data of the antibody tests. Data included sociodemographic details such as age, gender, ethnicity, place of work within the trust and actual occupation in terms of healthcare groups (eg doctors, nurses, therapists and administrative workers). Simple descriptive statistical methods were used to examine the prevalence rates of the antibodies within various sociodemographic groups. Simple statistical chi-squared tests of distribution were performed using the Stata statistical package.

\section{Results}

Out of 7,013 staff members, 6,212 (89\%) undertook the antibody test during the study period. Eighty per cent of them had had their tests within the first 2 weeks of the study period. There were no members that consented for the test who were not able to have the antibody test. The overall detection rate of IgG SARS-CoV-2 antibody was 26\% (1,584/6,212; Table 1; supplementary material S1).

The data was analysed according to various socio-demographic factors including age, gender, ethnicity, professional groups and geographical areas of work within the hospital. Univariate analyses reveal that there were no differences in the prevalence

Table 1. Socio-demographic features of prevalence of SARS-CoV-2 antibody in healthcare workers

\begin{tabular}{|c|c|c|c|c|}
\hline Socio-demographic factors & $\begin{array}{l}\text { Workforce headcount, } \\
\text { n (\% of total) }\end{array}$ & $\begin{array}{l}\text { Number of staff } \\
\text { tested, } n(\%)\end{array}$ & $\begin{array}{l}\text { SARS-CoV-2 antibody } \\
\text { detected, } n(\%)\end{array}$ & $\begin{array}{l}\text { Chi-squared test } \\
\text { of distribution }\end{array}$ \\
\hline Total & $7,013(100)$ & $6,212(89)$ & $1,584(26)$ & \\
\hline \multicolumn{5}{|l|}{ Ethnicity } \\
\hline White & $3,228(46)$ & $2,618(81)$ & $471(18)$ & \multirow{2}{*}{$\mathrm{p}<0.001$} \\
\hline BAME total & $3,671(52)$ & $2,799(76)$ & $894(32)$ & \\
\hline \multicolumn{5}{|l|}{ Professional groups } \\
\hline Medical & $948(14)$ & $797(84)$ & $182(23)$ & \multirow[t]{5}{*}{$p<0.001$} \\
\hline Nursing and midwifery & $2,439(35)$ & $1,961(80)$ & $554(28)$ & \\
\hline Allied clinical services & $1,866(27)$ & $1,429(77)$ & $396(28)$ & \\
\hline Healthcare scientists & $3,86(5)$ & $320(83)$ & $70(22)$ & \\
\hline Administrative and ancillary & $1,374(20)$ & $994(72)$ & $190(19)$ & \\
\hline \multicolumn{5}{|l|}{ Geographical area of work } \\
\hline ED, ITU and anaesthetics & $816(12)$ & $698(86)$ & $159(23)$ & \multirow[t]{5}{*}{$\mathrm{p}<0.001$} \\
\hline General wards & $1,310(19)$ & $953(73)$ & $405(43)$ & \\
\hline Theatre and site managers & $440(6)$ & $348(79)$ & $91(26)$ & \\
\hline Other clinical teams & $3,651(52)$ & $2,924(80)$ & $616(21)$ & \\
\hline Non-clinical areas & $796(11)$ & $578(73)$ & $121(21)$ & \\
\hline
\end{tabular}

BAME = black, Asian and minority ethnic; $E D=$ emergency department; ITU = intensive treatment unit. 
rates in terms of gender or age ( $p=n s)$. Compared with white staff members (18\%), prevalence of the antibody was significantly greater in black (38\%) and South Asian (27\%) staff members. The combined prevalence for all black, Asian or minority ethnic (BAME) staff members (black, South and East Asian or mixed race) was $32 \%(p<0.001)$.

The prevalence rates of staff in general wards $(43 \%)$ were significantly higher than other areas of the trust $(p<0.001)$. For staff in emergency medicine, intensive care and anaesthetics, the prevalence was $23 \%$, whereas for other clinical teams it was $21 \%$. As might be expected, for non-clinical staff the prevalence was also low at $21 \%$. In terms of professional groups, the prevalence rates were highest among nursing and allied clinical services ( $28 \%)$, followed by doctors $(23 \%)$, whereas, it was lower for nonclinical staff $(19 \% ; p<0.001)$

\section{Discussion}

This is the largest multi-ethnic hospital-based study that has described the prevalence of recent exposure to SARS-CoV-2 infection among healthcare workers as determined by presence of antibodies to the virus. The study has further examined associations of this prevalence in terms of various sociodemographic factors. Overall, the prevalence rate among this multi-ethnic cohort was $26 \%$.

There have been few studies that have described the prevalence of SARS-CoV-2 infection in recent months. A relatively small Dutch study $(1,353 / 9,705$ were tested) reported the rate to be $1 \%$, but this study was based on tests on symptomatic workers with a direct reverse transcriptase polymerase chain reaction test of oropharyngeal samples. ${ }^{9}$ Another observational cohort study showed healthcare workers to be nearly 12 times more likely to develop COVID-19 than the general population after multivariate adjustment (prevalence rates of $3.96 \%$ vs $0.33 \%$ ). ${ }^{6} \mathrm{~A}$ Chinese study reported $17 \%$ seroprevalence in 105 healthcare workers. ${ }^{8}$ A UK-based study reported $3 \%$ prevalence among its healthcare workers, albeit using SARS-CoV-2 viral antigen test. ${ }^{\text {? }}$

The strengths of this cross-sectional study include a large number of healthcare workers assessed within a very short period of time, thereby giving a good indication of prevalence within the cohort; a multi-ethnic diversity of the cohort which implies the rates could be generalisable to other healthcare populations; that the study was able to examine various socio-demographic factors associated with the prevalence of the infection which would enable healthcare institutions in terms of future workforce planning and appropriate allocation of personal protective equipment (PPE); that despite the test being entirely voluntary, nearly $90 \%$ of the potential workers consented to the test; and the reported high sensitivity (99\%) and specificity (100\%) of the antibody test. ${ }^{5,13}$

We also examined socio-demographic associations of SARSCoV-2 seroprevalence among healthcare workers. We found age and gender were not associated with SARS-CoV-2 exposure. However, we found the prevalence to be significantly higher among healthcare workers who were from BAME. While the workforce proportion is comparable (white $46 \%$ vs BAME $52 \%$; $p=n s), 32 \%$ of BAME were positive compared with $18 \%$ of white. There are possible speculative hypotheses that may explain this difference: ethnic genetic variability in terms of innate immune response to viral infections though yet unproven in SARS-CoV-2 infection specifically; higher prevalence of underlying health diseases such as diabetes which may lend them to be more susceptible to developing infections; higher proportion of BAME workers employed as frontline (public facing, service-based) workers who would be more exposed to patients with COVID-19; and worse deprivation rates and higher household density among the BAME population..$^{14-19}$ While further studies are needed to explore this difference further, it is important for healthcare institutions to conduct comprehensive COVID-19 risk assessments for all healthcare workers in light of this study, particularly for those from the BAME community to ensure that their future risk of exposure to SARS-CoV-2 is minimised.

As might be expected, this study confirmed that those professional groups that were high-risk workers (nurses and allied clinical services, $28 \%$ ) had a significantly higher prevalence rates than others such as doctors (23\%) and administrative workers (19\%). ${ }^{16-18}$ A marked difference was observed between those working in general wards ( $43 \%$ ) compared with those working in emergency medicine, intensive care or anaesthetics (23\%). Age, gender and ethnic proportions of workers in these areas were comparable. We hypothesise three possible reasons for this difference. First of all, patients with COVID-19 were likely to spend significantly more time in wards than in other areas, such as emergency medicine or intensive care, thus potentially leading to greater viral exposure to healthcare workers in these areas. Secondly, during the initial phase of the pandemic, there was a lot of uncertainty in terms of both national guidance and individual awareness in terms of what PPE was appropriate for various settings within the hospital. The disease was novel to all healthcare workers, and it took some time for clarity and a more structured approach to be developed in managing the balance between looking after patients with COVID-19 and individual risk of exposure. While workers in intensive care and relevant areas of emergency medicine were always advised to don full PPE, there was constant uncertainty and variation in national guidance regarding use of PPE in other areas. Finally, healthcare workers in general wards may often have been dealing with patients who had initially been deemed not to have COVID-19, but were found to have COVID-19 subsequently. Speculatively, one could have been less vigilant about social distancing and appropriate PPE than one would have done so if dealing with a confirmed COVID-19 patient. With time and development of better understanding, national guidance now advises healthcare workers to be vigilant regarding SARS-CoV-2 exposure at all times. ${ }^{20}$

There are some limitations to this study. Due to the design of the study, we could not examine any clinical features, including previous and current COVID-19 symptoms or presence of other clinical illnesses. We could not conduct any multivariable analyses to control for any confounding factors that may explain some of the results of this study. There were about $10 \%$ of workers whose ESR could not be matched with their antibody test information. However, it is unlikely that this missing data would have made any significant difference to the associations of prevalence rates identified in the study. The relevance of presence or absence of the antibody to future immunity is also unknown. Nevertheless, this study provides information that may be useful in future studies examining the role of antibody testing both in general populations as well as in healthcare settings. If future studies show that the immunity is lasting, it may also help in stratifying workforces and patients. 


\section{Conclusion}

This large multi-ethnic hospital-based study has described the prevalence of recent exposure to SARS-CoV-2 infection among healthcare workers and determined socio-demographic associations of this prevalence including ethnicity, professional healthcare groups and geographical areas of work in healthcare settings. The study provides information that may be useful in future COVID-19 studies examining the role of antibody testing both in general populations as well as in healthcare settings.

\section{Supplementary material}

Additional supplementary material may be found in the online version of this article at www.rcpjournals.org/clinmedicine: S1 - Fuller dataset of socio-demographic features of prevalence of SARS-CoV-2 antibody in healthcare workers.

\section{Conflicts of interest}

Dr Mehool Patel is on the editorial board of Clinical Medicine.

\section{References}

1 Huang C, Wang Y, Li X et al. Clinical features of patients infected with 2019 novel coronavirus in Wuhan, China. Lancet 2020;395: 497-506.

2 Guan WJ, Ni ZY, Hu Y et al. Clinical characteristics of coronavirus disease 2019 in China. N Engl J Med 2020;382:1708-20.

3 La Marca A, Capuzzo M, Paglia T et al. Testing for SARS-CoV-2 (COVID-19): a systematic review and clinical guide to molecular and serological in-vitro diagnostic assays. Reprod Biomed Online 2020;41:483-99.

4 Bastos ML, Tavaziva G, Abidi SK et al. Diagnostic accuracy of serological tests for COVID-19: systematic review and meta-analysis. BMJ 2020;370:m2516.

5 Petherick A. Developing antibody tests for SARS-CoV-2. Lancet 2020;395:1101-2.

6 Nguyen LH, Drew DA, Joshi AD et al. Risk of COVID-19 among frontline healthcare workers and the general community: a prospective cohort study. Lancet Public Health 2020;5:e475-83.

7 Rivett L, Sridhar S, Sparkes D et al. Screening of healthcare workers for SARS-CoV-2 highlights the role of asymptomatic carriage in COVID-19 transmission. elife 2020;9:e58728.

8 Chen Y, Tong X, Wang J et al. High SARS-CoV-2 antibody prevalence among healthcare workers exposed to COVID-19 patients. J Infect 2020;81:420-6.

9 Kluytmans-van den Bergh MFQ, Buiting AGM, Pas SD et al. Prevalence and clinical presentation of health care workers with symptoms of coronavirus disease 2019 in 2 Dutch hospitals during an early phase of the pandemic. JAMA Netw Open 2020:3:e209673.

10 Luo Y, Trevathan E, Qian Z et al. Asymptomatic SARS-CoV-2 infection in household contacts of a healthcare provider, Wuhan, China. Emerg Infect Dis. 2020;26:1930-3.

11 Weissman DN, de Perio MA, Radonovich LJ. COVID-19 and risks posed to personnel during endotracheal intubation, JAMA 2020;323 2027-8.

12 Philip P, Powis S. Antibody testing programme roll out for NHS staff and patients. NHS, 2020. www.england.nhs.uk/coronavirus/wpcontent/uploads/sites/52/2020/05/antibody-testing-programmeletter-25-may-2020.pdf

13 Bryan A, Pepper G, Wener MH et al. Performance characteristics of the Abbott Architect SARS-CoV-2 IgG assay and seroprevalence in Boise, Idaho. J Clin Microbiol 2020;58:e00941-20.

14 Kenney AD, Dowdle JA, Bozzacco L et al. Human genetic determinants of viral diseases. Annu Rev Genet 2017;51:241-63.

15 Sambaturu N, Mukherjee S, López-García M et al. Role of genetic heterogeneity in determining the epidemiological severity of H1N1 influenza. PLoS Comput Biol 2018;14:e1006069.

16 Williamson E, Walker AJ, Bhaskaran K et al. OpenSAFELY: factors associated with COVID-19-related hospital death in the linked electronic health records of 17 million adult NHS patients. medRxiv 2020.05.06.20092999.

17 Lassale C, Gaye B, Hamer M, Gale CR, Batty GD. Ethnic disparities in hospitalisation for COVID-19 in England: The role of socioeconomic factors, mental health, and inflammatory and pro-inflammatory factors in a community-based cohort study. Brain Behav Immun 2020;88:44-9.

18 The Institute for Fiscal Studies. Are some ethnic groups more vulnerable to COVID-19 than others? IFS, 2020. www.ifs.org.uk/ inequality/chapter/are-some-ethnic-groups-more-vulnerable-tocovid-19-than-others

19 Aldridge RW, Lewer D, Katikireddi SV. Black Asian and minority ethnic groups in England are at increased risk of death from COVID-19: indirect standardisation of NHS mortality data. Wellcome Open Res 2020;5:88.

20 Public Health England, NHS England. COVID-19: infection prevention and control (IPC). PHE, 2020. www.gov.uk/government/ publications/wuhan-novel-coronavirus-infection-prevention-andcontrol/covid-19-personal-protective-equipment-ppe [Accessed 09 July 2020].

Address for correspondence: Dr Mehool Patel, University Hospital Lewisham, Lewisham and Greenwich NHS Trust, High Street, Lewisham, London SE13 6LH, UK. Email: mehool.patel@nhs.net 